\title{
Performance Analysis of MIMO-OFDM System Using Singular Value Decomposition and Water Filling Algorithm
}

\author{
Md. Noor-A-Rahim ${ }^{1}$, Md. Saiful Islam², Md. Nashid Anjum ${ }^{3}$, Md. Kamal Hosain ${ }^{4}$, and Abbas Z. Kouzani ${ }^{5}$ \\ ${ }^{1,3}$ Electronics \& Communications Engineering, Khulna University of Engineering and Technology \\ Khulna, Bangladesh. \\ ${ }^{4}$ Electronics and Telecommunications Engineering, Rajshahi University of Engineering and Technology \\ Rajshahi, Bangladesh-6204. \\ ${ }^{2,5}$ School of Engineering, Deakin University, Victoria 3217, Australia
}

\begin{abstract}
In this paper, MIMO is paired up with OFDM to improve the performance of wireless transmission systems. Multiple antennas are employed both at the transmitting as well as receiving ends. The performance of an OFDM system is measured, considering multipath delay spread, channel noise, Rayleigh fading channel and distortion. In this paper, bits are generated and then mapped with modulation schemes such as QPSK, 8PSK, and QAM. Then, the mapped data is divided into blocks of 120 modulated data where a training sequence of the data is inserted both at the beginning and ending parts of the block. The equalization is used to determine the variation to the rest of data. The singular value decomposition (SVD) and water filling algorithm have been employed to measure the performance of the MIMO-OFDM integrated systems. Therefore, the capacity is increased by transmitting different streams of data through different antennas at a same carrier frequency. Any intersymbol interference (ISI) produced after the transmission is recovered by using spatial sampling integrated with the signal processing algorithm. Furthermore, the performance remains the same with different combinations of transmitting and receiving antennas.
\end{abstract}

\section{Keywords-MIMO; OFDM; ISI; SVD; Water filling algorithm}

\section{INTRODUCTION}

Wireless communication systems face high level of ISI which originates from multipath propagation and inherent delay spread. A multicarrier based technique such as orthogonal frequency division multiplexing (OFDM) can be used for extenuating ISI to improve capacity and spectral efficiency $(\mathrm{bps} / \mathrm{Hz})$ in a wireless system [2]. In addition, MIMO systems are promising techniques to increase performance with acceptable bit error rate (BER) by using a number of antennas [3]. A MIMO-OFDM system transmits OFDM modulated data from multiple antennas at the transmitter. Data transmitted with subcarriers at different antennas are mutually orthogonal. The receiver extracts different data stream from different subcarriers after OFDM demodulation and MIMO decoding. Flat fading MIMO algorithms reduce computational requirement and make MIMO-OFDM attractive for mobile applications [1].

Although MIMO was first introduced in 1990s, it is still an active research area. Adaptive technology has been used in
MIMO systems to get high spectral efficiency. For a timevarying channel, we need a simple algorithm which adaptively adjusts transmit parameters for high capacity [4]. A novel power allocation and adaptive modulation algorithm for water filling can be created by combining the adaptive technology with cell planning, which can reduce interfere to the adjacent cell. Adaptive SVD algorithm uses a two-step recursive method, is an important technique to exploit the full capability of MIMO-OFDM systems. Adaptive power control based on water filling, and rate adaptation maximizes the spectral efficiency of MIMO-OFDM in discrete and upper bounded alphabets [5]. Whereas, Y. Jiang, et al. have investigated that two dimensional water filling power allocation algorithm with the SVD for MIMO-OFDM system provide higher capacity than that of its uniform power allocation counterpart with one dimensional water filling power allocation scheme [6].

Co-Channel Interference (CCI) between transmitted and received substreams of single-frame TDMA data can be minimized by OFDM-MIMO systems with V-BLAST signal processing at the receiver [7]. A custom hardware for computing SVD can reduce its computing time [8]. Generalized SVD based on beamforming can be used in MIMO broadcasting, and in MIMO relaying [9]. W. Liejun has proposed improved water filling algorithm for determining the optimal transmit powers and larger throughputs in MIMO-OFDM system [10].

This paper focuses on high data rate wireless communication due to such benefits as inter-symbol interference (ISI) free transmission, high spectral efficiency, and reduced equalization complexity. The paper shows that OFDM-MIMO system with SVD and water filling algorithm is robust against the signal-to-noise variations, and its performance is superior to a single antenna system.

\section{BASIC MIMO-OFDM SYSTEM}

Orthogonal frequency division multiplexing (OFDM) is a popular wireless multicarrier transmission technique. It is a promising candidate for next-generation wired and mobile wireless systems. The basic principle of OFDM is to split a high rate data stream into a number of lower rate streams so that the lower data rate can be transmitted simultaneously over 
a number of subcarriers. In OFDM, the amount of dispersion in time, caused by multipath delay spread, is decreased due to the increased symbol duration for lower rate parallel subcarriers. The spectrum of OFDM is more efficient because of the use of closer channels space. Interferences are prevented by making all the carriers orthogonal to one another. MIMO systems utilize space-multiplex by using antenna arrays to enhance the efficiency in the used bandwidth. These systems are defined by spatial diversity and spatial multiplexing. Spatial diversity is known as Rx- and Tx-diversity. Signal copies are transferred from another antenna, or received at more than one antenna. With spatial multiplexing, the system carries more than one spatial data stream over one frequency, simultaneously.

In an $\mathrm{N}$ subcarriers MIMO-OFDM system, the individual data streams are first passed through an OFDM modulator. Then, the resulting OFDM symbols are launched simultaneously through the transmit antennas. In the receiver side, the individual received signals are passed through OFDM demodulator. The outputs of the OFDM demodulator are decoded and rearranged to get the desired output. Fig. 1 shows the schematic diagram of a basic MIMO-OFDM system.

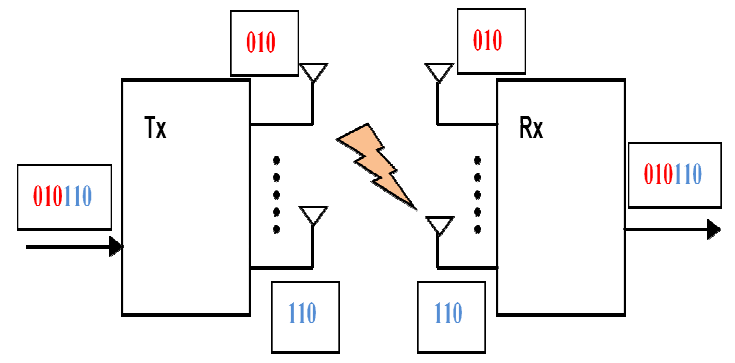

Figure 1. A basic MIMO-OFDM system [1].

\section{THEORY}

\section{A. Spatial Multiplexing}

The transmission of multiple data streams over more than one antenna is called spatial multiplexing [11]. The advantage of spatial multiplexing is linear capacity gains in relation to the number of transmit antennas.

\section{B. Spatial Diversity}

Spatial diversity improves the signal quality and achieves a higher signal to noise ratio at the receiver side. The principle of diversity relies on the transmission of structured redundancy. This redundancy can be transmitted at any time, from any antenna, over any frequency or at any polarization. Two kinds of spatial diversity need to be considered: (i)Tx-Diversity, where a signal copy is transmitted through more than one antenna, (ii) Rx-Diversity, where the received signal is multiple evaluated [12].

\section{MIMO Channel Matrix}

The MIMO system has multiple links and operates on the same frequency whereas the non-MIMO system is linked over multiple channels by several frequencies. The challenge of this technology is the separation and the equalization of the signal in all paths. The channel model includes the channel matrix $\mathrm{H}$ with the direct and the indirect channel components. The direct components represent the channel flatness whereas the indirect components stand for the channel isolation. Consider an OFDM symbol of $\mathrm{N}$ sub-symbols and cyclic prefix, $\mathrm{P}$, the length of which is less than the last significant tap delay. The sent signal is ' $x$ ' and the received signal is ' $y$ '. A time-invariant and narrowband channel is assumed. The output $\mathrm{y}$ can be expressed in a matrix format as follows [13]:

$$
\mathrm{y}=\mathrm{H} \times \eta
$$

where $\mathrm{y}$ is the received vector, $\mathrm{x}$ is the transmitted vector and $\eta$ is complex $A W G N$ vector.

\section{Capacity}

Claude Elwood Shannon [14] developed the following equation for the theoretical channel capacity:

$$
\mathrm{c}_{\text {SISO }}=\mathrm{f}_{\mathrm{g}} \log _{2}(1+\mathrm{S} / \mathrm{N})
$$

It includes the transmission bandwidth fg and the signal-tonoise ratio. Most channel capacity improvements are based on bandwidth extensions or modulations. The Shannon capacity of MIMO Systems additionally depends on the number of antennas. For MIMO the capacity is given by the following equation:

$$
\mathrm{c}_{\text {MIMO }}=\mathrm{Mf}_{\mathrm{g}} \log _{2}(1+\mathrm{S} / \mathrm{N})
$$

where $\mathrm{M}$ is the minimum of MT (number of transmitting antennas) or MR (number of receiving antennas) and represents the number of spatial streams. For example, a 2 x 3 system can only support two spatial streams, which is also true for a $2 \times 4$ system. Asymmetrical antennas constellations are referred to as receive or transmit diversity. In that case, the capacity $\mathrm{CTx} / \mathrm{Rx}$ grows logarithmically with the number of antennas $[15,16]$.

$$
\mathrm{C}_{\mathrm{Tx} / \mathrm{Rx}}=\mathrm{f}_{\mathrm{g}} \log _{2}\left(1+\mathrm{M}\left(\frac{\mathrm{s}}{\mathrm{N}}\right)\right)
$$

\section{E. SNR Threshold}

In the proposed transmission control scheme, the threshold value identifies whether multiple transmit antennas should be used or not. Therefore, it is obvious that selecting the right threshold value has significant effect on the performance of the MIMO system. A small threshold increases the complexity without achieving high multiplexing gain [17]. On the other hand, a large threshold causes loosing multiplexing gain due to not using the multiple antennas.

\section{F. Water Filling}

Water filling refers to a technique whereby the power for the spatial channels are adjusted based on the channels gain. The channel with high gain and signal to noise ratio (SNR) is given more power. More power maximizes the sum of data rates in all subchannels. The data rate in each subchannel is related to the power allocation by Shannon's Gaussian capacity formula $\mathrm{C}=\mathrm{B} \log (1+\mathrm{SNR})$. However, because of the capacity is a logarithmic function of power, the data rate is usually insensitive to the exact power allocation. This motivates the search for simpler power allocation schemes that can perform close to the optimal. The water filling algorithm is based on an iterative procedure, as described below [18].

Filling Algorithm: VEC is the absolute or relative level of noise in LINEAR units at different frequencies, space or 
whatever bins. PCON is a total power constrain given in the same units as the VEC. TOL is an acceptable tolerance in the units of VEC. WLINE indicates the WATERLINE level in units of VEC so that:

$$
\operatorname{abs}(\mathrm{PCON}-\mathrm{SUM}(\mathrm{MAX}(\mathrm{WLINE}-\mathrm{VEC}, 0)))<=\text { TOL. }
$$

The algorithm is built in such a way that PCON $>=$ SUM (MAX (WLINE - VEC, 0)) and never PCON < SUM (MAX (WLINE - VEC, 0)). VEC must be a row vector representing a noise level. PCON and TOL must be scalars in the same units as VEC.

(i) Find the length $(\mathrm{N})$ of VEC. $\mathrm{N}=$ length (vec)

(ii) Determine the initial waterline level, WLINE. wline $=\min (\mathrm{vec})+\mathrm{pcon} / \mathrm{N}$

(iii) Measure the total power of current waterline. ptot $=\operatorname{sum}(\max ($ wline - vec, 0$))$

(iv) Repeat step (a) \& (b) until

abs (pcon - ptot) $>$ tol
a) wline $=$ wline $+($ pcon - ptot $) / \mathrm{N}$

b) $\operatorname{ptot}=\operatorname{sum}(\max ($ wline - vec, 0$))$

The water filling algorithm is shown in fig. 2 .

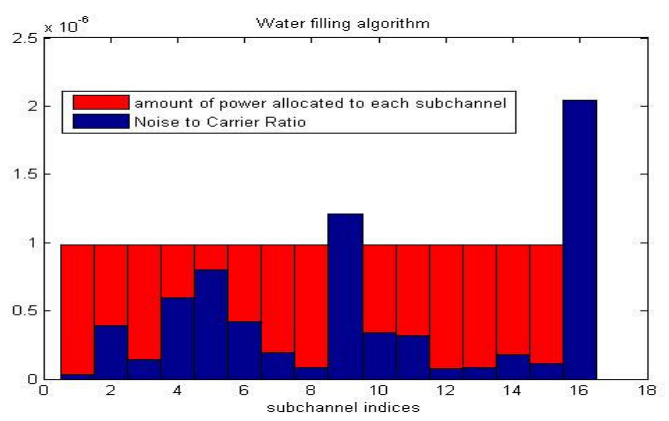

Figure 2. Water filling algorithm. where $U$ and $V$ are unitary matrices and $V^{\mathrm{h}}$ is the hermitian of $\mathrm{V}$. U has dimension of R $x \mathrm{R}$ and $\mathrm{V}$ has dimension of T $\mathrm{x}$ T. $\sum$ is a $\mathrm{T} x \mathrm{R}$ matrix. If $\mathrm{T}=\mathrm{R}$, then $\sum$ become a diagonal matrix. If $\mathrm{T}>\mathrm{R}$, it is made of $\mathrm{R} \times \mathrm{R}$ diagonal matrix followed by $\mathrm{T}-\mathrm{R}$ zero columns. If $\mathrm{T}<\mathrm{R}$, it is made of $\mathrm{T} \times \mathrm{T}$ diagonal matrix followed by $\mathrm{R}-\mathrm{T}$ zero rows. This operation is called the singular value decomposition of $\mathrm{H}[9,19]$.

In case, where $T \neq R$, the number of spatial channels become restricted to the minimum of $\mathrm{T}$ and $\mathrm{R}$. If the number of transmit antennas is greater than the receive antennas $(T>R)$, $\mathrm{U}$ will be an $\mathrm{R} \times \mathrm{R}$ matrix, $\mathrm{V}$ will be a T x T matrix and $\sum$ will be made of a square matrix of order $\mathrm{R}$ followed by $\mathrm{T}-\mathrm{R}$ zero columns $[9,19]$.

\section{MODELLING AND SIMULATION}

\section{A. OFDM Simulation Model}

The OFDM system is modeled using MATLAB and is shown in Fig. 3[20]. The data to be transmitted on each carrier is differentially encoded with previous symbols, then mapped into a Phase Shift Keying (PSK) format. The data on each symbol is then mapped to a phase angle based on the modulation method such as QPSK. The serial data stream is formatted into the word size required for transmission, e.g. 2 bits/word for QPSK, and shifted into a parallel format. Zero padding has used in our system to increase sampling rates for better resolution of signals. After the required spectrum is worked out, an inverse Fourier transform is performed to find the corresponding time waveform. The guard period is then added to the start of each symbol. After the guard has been added, the symbols are then converted back to a serial time waveform. This is then the base band signal for the OFDM transmission.

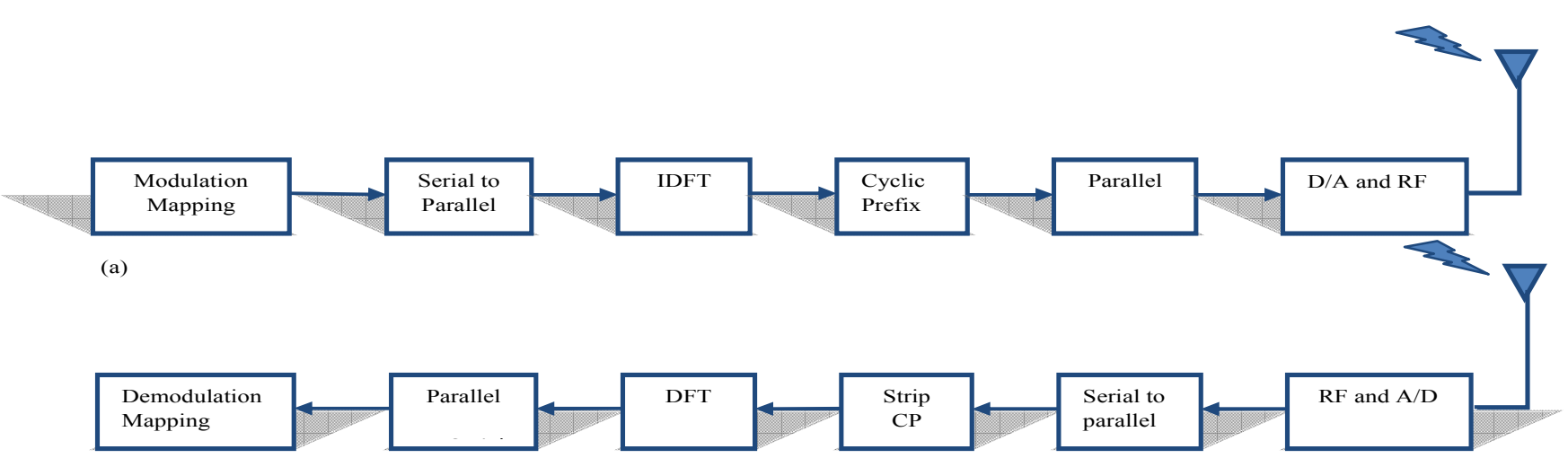

(b)

Figure 3. OFDM model used for simulations: (a) OFDM transmitter and (b) OFDM receiver

\section{G. Singular Value Decomposition}

The SVD technique decouples the channel matrix in spatial domain in a way similar to the DFT decoupling the channel in the frequency domain. The channel matrix $\mathrm{H}$ is the $\mathrm{T} \times \mathrm{R}$ channel matrix. If $\mathrm{H}$ has independent rows and columns, SVD yields:

$$
\mathrm{H}=\mathrm{U} \sum \mathrm{V}^{\mathrm{h}}
$$

A channel model is then applied to the transmitted signal. The model allows for the signal to noise ratio (SNR), multipath, and peak power clipping to be controlled. The SNR is set by adding a known amount of white noise to the transmitted signal. Multipath delay spread is then added by simulating the delay spread using an FIR filter. The length of the FIR filter represents the maximum delay spread, while the coefficient amplitude represents the reflected signal magnitude. 
The receiver basically does the reverse operation to the transmitter. In the receiving side, the model recovers the input data, and performs an analysis to determine the transmission error rate. Table I represents the OFDM system parameters used for the simulation.

TABLE I. OFDM SYSTEM PARAMETERS USED FOR SIMULATION

\begin{tabular}{|c|c|}
\hline Parameter & Value \\
\hline Carrier Modulation & QPSK \\
\hline FFT Size & 128 \\
\hline Number of Carriers & 120 \\
\hline Guard Time & 32 samples (25\%) \\
\hline Guard Period Type & Full cyclic extension of the symbol \\
\hline
\end{tabular}

\section{B. OFDM Simulation Result}

The simulation model accepts inputs as text or audio files, binary, sinusoidal, or random data. The channel simulation allows examination of common wireless multipath channel characteristics such Rayleigh fading channel with various Doppler Effect. Fig. 4 represents data streams after modulation mapping (at transmission end). Fig. 5 shows amplitude and phase spectrum of transmitted OFDM signal. Fig. 6 and Fig. 7 depict amplitude and phase spectrum of received OFDM signal with Dophlar spread 15 and 90 respectively. And, Fig. 8 illustrates the received signal spectrum after FFT.

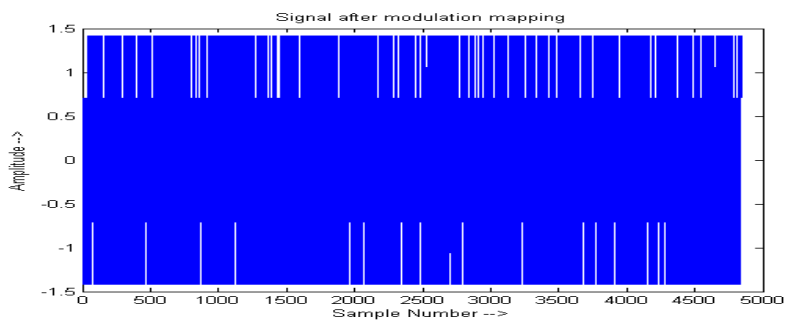

Figure 4. Data streams after modulation mapping (At transmission end).
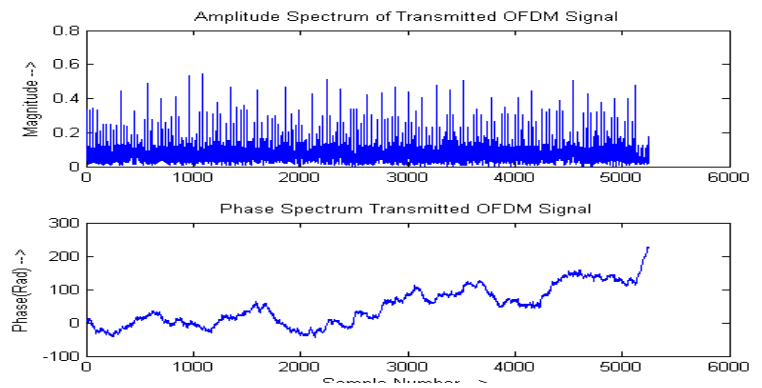

Amplitude Spectrum of Received OFDM Signal

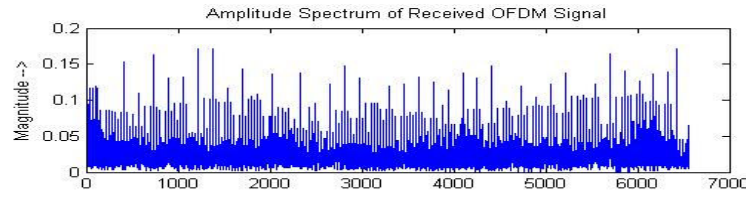

Phase Spectrum Received OFDM Signal

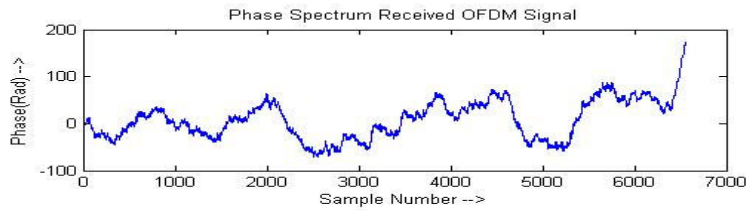

Figure 6. Amplitude and phase spectrum received OFDM signal (Dophlar spead $=15$ ).
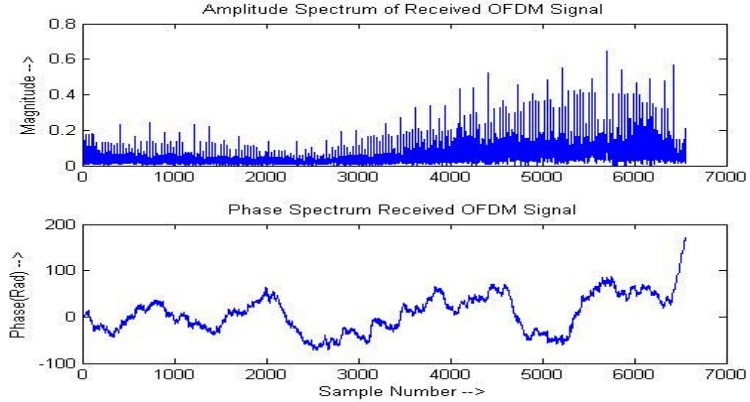

Figure 7. Amplitude and phase spectrum received OFDM signal (Dophlar
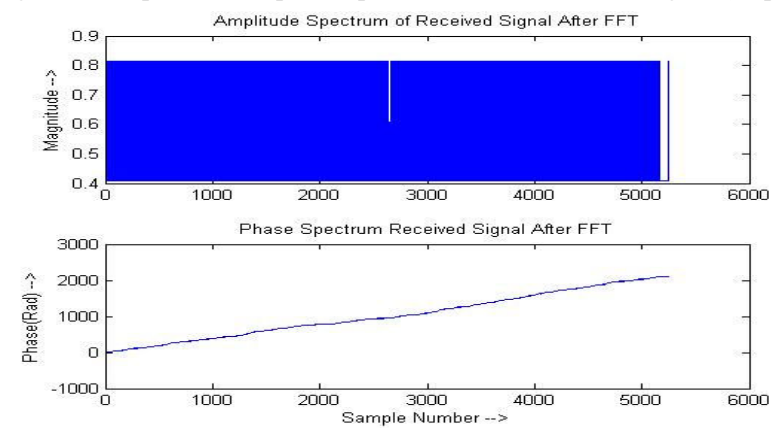

Figure 8. Received signal spectrum after FFT.

\section{MIMO-OFDM Simulation Modelling}

In this simulation, a highly scattered environment is considered. The capacity of a MIMO channel is analyzed with the antenna configuration as shown in Table II. Each channel is considered as a parallel flat fading channel. The power in a parallel channel (after decomposition) is distributed as water filling algorithm. Channel matrix $\mathrm{H}$ is measured using Rayleigh distribution function. This simulation computes channel capacity and PDF (probability density function) of elements in SVD of matrix $\mathrm{H}$, by varying the SNR from $-10 \mathrm{~dB}$ to $20 \mathrm{~dB}$, where 104 iterations are performed.

TABLE II. TRANSMITTING AND RECEIVING ANTENNA COMBINATION

\begin{tabular}{|c|c|c|}
\hline Combination & No. of tx antenna & No. of rx antenna \\
\hline 1 & 1 & 1 \\
\hline 2 & 2 & 2 \\
\hline 3 & 2 & 3 \\
\hline 4 & 3 & 2 \\
\hline 5 & 4 & 4 \\
\hline
\end{tabular}

\section{MIMO-OFDM Simulation Results}

The channel capacity vs SNR curve of different MIMO systems is shown in Fig. 9. Fig. 10 represents the power spectral density (PDF) vs SNR. These graphs depict that the 4 $\mathrm{x} 4$ MIMO systems provides better channel capacity and PDF than other combinations. This indicates that a higher order MIMO system increases the system performance. It is interesting to note that the system performance remains almost the same when the number of transmitter and receiver antennas is altered (2x3 MIMO and 3x2 MIMO systems). 


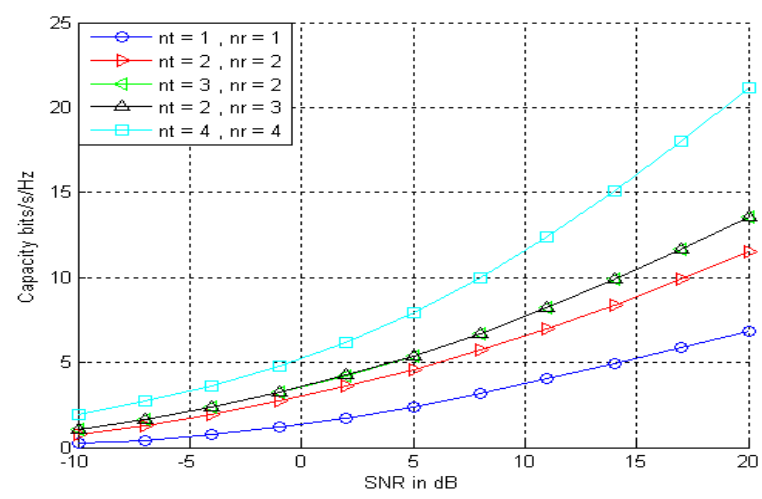

Figure 9. Comparison of channel capacity of different MIMO system.

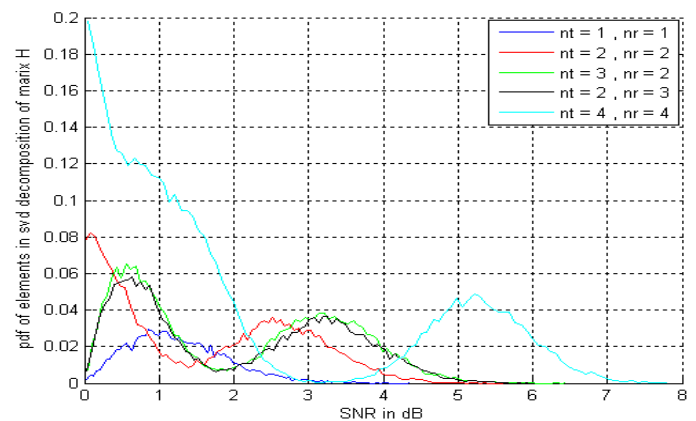

Figure 10. Comparison of PDF of different MIMO system.

\section{CONCLUSION}

An OFDM link was demonstrated through computer simulations and practical tests performed on a low bandwidth base-band signal. Four main performance criteria were tested, which include OFDM's tolerance to multipath delay spread, channel noise, peak power clipping and start time error. Several other important factors affecting the performance of OFDM have only been partly measured. In the past, there were a lot of problems with multiple wave propagation which led to creation of ISI. The MIMO has helped to reduce the ISI problem. With the implementation of MIMO-OFDM, the probability that the transmission arrives at the receiver with little or no error is greatly increased compared to the rest of the transmission techniques. In this system, the capacity is increased significantly by transmitting the different streams of data through different antennas at a same carrier frequency.

\section{REFERENCES}

[1] H. Bolcskei, "MIMO-OFDM wireless systems: basics, perspectives, and challenges," Wireless Communications, IEEE, vol. 13, pp. 31-37, 2006.

[2] R. Rao, "Impact of Phase Noise in MIMO-OFDM Systems," 2007.

[3] Y. Li and N. Sollenberger, "Adaptive antenna arrays for OFDM systems with cochannel interference," Communications, IEEE Transactions on, vol. 47,pp. 217-229, 2002.

[4] A. Goldsmith and S. Chua, "Variable-rate variable-power MQAM for fading channels," Communications, IEEE Transactions on, vol. 45, pp. 1218-1230, 2002.

[5] J. Huang and S. Signell, "The application of rate adaptation with finite alphabet in MIMO-OFDM," 2005, pp. 946-949.

[6] Y. Jiang, et al., "Two-dimensional water-filling power allocation algorithm for MIMO-OFDM systems," SCIENCE CHINA Information Sciences, vol. 53, pp. 1242-1250, 2010.

[7] E. Whu, "MIMO-OFDM Systems for High Data Rate Wireless Networks," Proj. Report for EE360 Advanced Wireless Networks: MIMO-OFDM Wireless Networks.

[8] Y. Wang, et al., "Singular Value Decomposition hardware for MIMO: State of the art and custom design," Cancun, 2010, pp. 400-405.

[9] D. Senaratne and C. Tellambura, "Generalized singular value decomposition for coordinated beamforming in MIMO systems," Miami, FL, 2010.

[10] W. Liejun, "An Improved Water-filling Power Allocation Method in MIMO OFDM Systems," Information Technology Journal, vol. 10, pp. 639-647, 2011.

[11] A. Gorokhov, et al., "Receive antenna selection for MIMO spatial multiplexing: theory and algorithms," Signal Processing, IEEE Transactions on, vol. 51, pp. 2796-2807, 2005.

[12] V. R. Anreddy and M. A. Ingram, "Capacity of measured ricean and rayleigh indoor MIMO channels at $2.4 \mathrm{GHz}$ with polarization and spatial diversity," in Wireless Communications and Networking Conference, 2006. WCNC 2006. IEEE, 2006, pp. 946-951.

[13] S. Venkatesan, et al., "Capacity of a Gaussian MIMO channel with nonzero mean," 2004, pp. 1767-1771.

[14] T. Kan, et al., "MIMO Channel Capacity of a Measured Radio Channel for Outdoor Macro Cellular Systems at 3GHz-Band," in Vehicular Technology Conference Fall (VTC 2009-Fall), 2009 IEEE 70th, 2009, pp. 1-5.

[15] S. Sumei, et al., "Precoding for Asymmetric MIMO-OFDM Channels," in Communications, 2006. ICC '06. IEEE International Conference on, 2006, pp. 3117-3122.

[16] J. Wang, et al., "Capacity of MIMO-OFDM system with multi-path fading channel," Beijing, 2009.

[17] A. Maaref and S. Aissa, "Combined adaptive modulation and truncated ARQ for packet data transmission in MIMO systems," 2005, pp. 38183822 .

[18] G. Scutari, et al., "The MIMO iterative waterfilling algorithm," Signal Processing, IEEE Transactions on, vol. 57, pp. 1917-1935, 2009.

[19] G. Golub and C. Reinsch, "Singular value decomposition and least squares solutions," Numerische Mathematik, vol. 14, pp. 403-420, 1970.

[20] I. Khan, et al., "Capacity and performance analysis of space-time block coded MIMO-OFDM systems over Rician fading channel," International Journal of Electrical and Computer Engineering, 2009. 